\title{
Regulation of myo-inositol biosynthesis by p53-ISYNA1 pathway
}

\author{
TOMOYUKI KOGUCHI $^{1,3}$, CHIZU TANIKAWA $^{2}$, JINICHI MORI ${ }^{1}$, YOSHIYUKI KOJIMA $^{3}$ and KOICHI MATSUDA $^{1}$ \\ ${ }^{1}$ Laboratory of Clinical Sequence, Department of Computational Biology and Medical Sciences, \\ Graduate School of Frontier Sciences, ${ }^{2}$ Laboratory of Molecular Medicine, Human Genome Center, \\ Institute of Medical Science, The University of Tokyo, Tokyo; ${ }^{3}$ Department of Urology, \\ Fukushima Medical University School of Medicine, Fukushima, Japan
}

Received January 22, 2016; Accepted February 25, 2016

DOI: $10.3892 /$ ijo.2016.3456

\begin{abstract}
In response to various cellular stresses, p53 exerts its tumor suppressive effects such as apoptosis, cell cycle arrest, and senescence through the induction of its target genes. Recently, p53 was shown to control cellular homeostasis by regulating energy metabolism, glycolysis, antioxidant effect, and autophagy. However, its function in inositol synthesis was not reported. Through a microarray screening, we found that five genes related with myo-inositol metabolism were induced by p53. DNA damage enhanced intracellular myo-inositol content in HCT116 p53 $53^{+/+}$cells, but not in HCT116 p53/- cells. We also indicated that inositol 3-phosphate synthase (ISYNA1) which encodes an enzyme essential for myo-inositol biosynthesis as a direct target of p53. Activated p53 regulated ISYNA1 expression through p53 response element in the seventh exon. Ectopic ISYNA1 expression increased myo-inositol levels in the cells and suppressed tumor cell growth. Knockdown of ISYNA1 caused resistance to adriamycin treatment, demonstrating the role of ISYNA1 in p53-mediated growth suppression. Furthermore, ISYNAI expression was significantly associated with p53 mutation in bladder, breast cancer, head and neck squamous cell carcinoma, lung squamous cell carcinoma, and pancreatic adenocarcinoma. Our findings revealed a novel role of p53 in myo-inositol biosynthesis which could be a potential therapeutic target.
\end{abstract}

\section{Introduction}

p53 is one of the most frequently mutated tumor suppressor genes (1,2). In response to various cellular stresses, ATM-Chk2 cascade stabilizes p53 protein through the phosphorylation of its N-terminal domain (3). Activated p53 functions as a transcription factor and exerts its tumor suppressive effects such as

Correspondence to: Professor Koichi Matsuda, Laboratory of Clinical Sequence, Department of Computational Biology and Medical Sciences, Graduate School of Frontier Sciences, The University of Tokyo, 4-6-1 Shirokanedai, Minato, Tokyo 108-8639, Japan

E-mail: koichima@ims.u-tokyo.ac.jp

Key words: p53, inositol 3-phosphate synthase, myo-inositol apoptosis, cell cycle arrest, and senescence through the induction of its target genes $(1,2)$. In addition to genes related with cell proliferation, regulation of glycolysis (4), energy metabolism, antioxidant effect (5), autophagy (6), and respiration with mitochondria are reported as novel functions of p53. Thus, p53 regulates not only tumor cell growth but also pathways related with cellular homeostasis. Since inactivation of p53 is the most common feature of cancer cells, the elucidation of p53 signaling pathways would contribute to the understanding of tumor cells as well as for drug development.

Myo-inositol is water-soluble vitamin found in a variety of food products, and are also synthesized in cells (7). Previous studies indicated that myo-inositol has various functions including glucose and lipid metabolism $(8,9)$, neurotropic effect (10), and tumor suppression (11-13). However, the regulation of myo-inositol biosynthesis in cancer tissues has not been disclosed yet. Through a cDNA microarray screening using mRNAs isolated from HCT116 $p 53^{+/+}$and HCT116 $p 53^{-/-}$cells, we identified ISYNAI which encodes an enzyme essential for myo-inositol biosynthesis as a novel p53 target.

\section{Materials and methods}

cDNA microarray. Gene expression analysis was performed using SurePrint G3 Human GE 8x60K microarray (Agilent, Santa Clara, CA, USA) according to the manufacturer's protocol. Briefly, HCT116 $p 53^{+/+}$or HCT116 $p 53^{-/-}$cells were treated with $2 \mu \mathrm{g} / \mathrm{ml}$ of adriamycin (ADR) for $2 \mathrm{~h}$ and incubated at $37^{\circ} \mathrm{C}$ until harvest. At 12,24 and $48 \mathrm{~h}$ after treatment, total RNA was isolated from the cells using standard protocols. Each RNA sample was labeled and hybridized to array slides.

Cell culture and treatment. Human embryonic kidney cells HEK293T were obtained from Riken Cell Bank. Human cancer cell lines U373MG (astrocytoma), HepG2 (hepatocellular carcinoma), and HCT116 (colorectal adenocarcinoma) were purchased from American Type Culture Collection. HCT116 $p 53^{+/+}$and HCT116 $p 53^{-/-}$cells lines were gifts from B. Vogelstein (Johns Hopkins University, Baltimore, MD, USA). HEK293T, HCT116, and HepG2 cells were transfected with plasmids using FuGENE 6 (Promega, Madison, WI, USA). U373 MG cells were transfected with plasmids using FuGENE 6 or Lipofectamine LTX (Invitrogen, Carlsbad, CA, USA). Small interfering RNA (siRNA) oligonucleotides, 
commercially synthesized by Sigma Genosys, were transfected with Lipofectamine RNAiMAX reagent (Invitrogen). Sequences of siRNA oligonucleotides are shown in Table I. We generated and purified replication-deficient recombinant viruses expressing p53 (Ad-p53) or LacZ (Ad-LacZ) as described previously (14). U373MG (p53-mutant) cells were infected with viral solutions at various amounts of multiplicity of infection (MOI) and incubated at $37^{\circ} \mathrm{C}$ until the time of harvest. For treatment with genotoxic stress, cells were incubated with $2 \mu \mathrm{g} / \mathrm{ml}$ of ADR for $2 \mathrm{~h}$.

Plasmid construction. The entire coding sequence of ISYNAI isoforms 1 and 4 were amplified by PCR using KOD-Plus DNA polymerase (Toyobo, Osaka, Japan), and inserted into the $E c o R V$ and $\mathrm{XhoI}$ sites of pCAGGS vector. ISYNA1 isoform 2 expression vector was constructed by site-directed mutagenesis using ISYNA1 isoform 1 as a template. The construct was confirmed by DNA sequence analysis. Primers are shown in Table I.

Quantitative real-time PCR. Total RNA was isolated from human cells and mouse tissues using RNeasy Plus Mini kits (Qiagen, Valencia, CA, USA) and RNeasy Plus Universal Mini kits (Qiagen) according to the manufacturer's instructions. Complementary DNAs were synthesized using Super Script III reverse transcriptase (Invitrogen). Quantitative real-time PCR (qPCR) was conducted using SYBR Green Master Mix on a Light Cycler 480 (Roche, Basel, Switzerland). Primer sequences are shown in Table I.

Western blot analysis. To prepare whole cell extracts, cells were collected and lysed in chilled RIPA buffer $(50 \mathrm{mmol} / \mathrm{l}$ Tris- $\mathrm{HCl}$ at $\mathrm{pH} 8.0,150 \mathrm{mmol} / 1 \mathrm{NaCl}, 0.1 \% \mathrm{SDS}, 0.5 \%$ sodium deoxycholate, and 1\% NP40) containing $1 \mathrm{mM}$ phenylmethylsulphonyl fluoride (PMSF), $0.1 \mathrm{mM}$ DTT and $0.1 \%$ Calbiochem Protease Inhibitor Cocktail Set III, EDTA-Free (EMD Chemicals Inc., Merck KGaA, Darmstadt, Germany). Samples were sonicated for $15 \mathrm{~min}$ with a $30 \mathrm{sec}$ on/30 sec off cycle using Bioruptor UCD-200 (Cosmobio, Tokyo, Japan). After centrifugation at $16,000 \mathrm{x}$ g for $15 \mathrm{~min}$, supernatants were collected and boiled in SDS sample buffer (Bio-Rad, Hercules, CA, USA). SDS-polyacrylamide gel electrophoresis (SDS-PAGE) was performed for each sample, and the proteins were then transferred to a nitrocellulose membrane (Hybond $^{\mathrm{TM}}$ ECL $^{\mathrm{TM}}$, Amersham, Piscataway, NJ, USA). Protein bands on western blots were visualized by chemiluminescent detection (ECL, Amersham and Immobilon, Millipore). Anti$\beta$-actin monoclonal antibody (AC-15) was purchased from Abcam (Cambridge, UK). Anti-ISYNA1 monoclonal antibody (sc-271830) and anti-p53 monoclonal antibody were purchased from Santa Cruz Biotechnology (Santa Cruz, CA, USA). Antip2 $1^{\mathrm{WAF} 1}$ monoclonal antibody (OP64) was purchased from Merck Millipore (Darmstadt, Germany).

Immunocytochemical analyses. Cells were seeded on coverslips in 24-well plates. After each treatment indicated in the text, cells were washed in phosphate-buffered saline (PBS) before fixation in $4 \%$ paraformaldehyde. Cells were immunostained overnight with primary antibodies followed by incubation with Alexa Fluor 488-conjugated secondary
IgG (Molecular Probes) for $1 \mathrm{~h}$. Cells were subjected to 4'-6-diamidino-2-phenylindole (DAPI) staining to visualize cell nuclei. Immunofluorescence was visualized and recorded on an Olympus FV1000D laser confocal microscope. Images were processed using Olympus FV10-ASW software and Adobe Photoshop CS3.

Gene reporter assay. DNA fragments, including the potential p53-response elements (REs), were amplified and subcloned into the pGL4.24 vector (Promega). Point mutations ' $T$ ' were inserted at the 4th and the 14th nucleotide ' $\mathrm{C}$ ' and the 7th and the 17th nucleotide ' $G$ ' of each RE by site-directed mutagenesis. Reporter assays were performed using the Dual Luciferase assay system (Promega) as described previously (15). Primers for amplification and mutagenesis are shown in Table I.

Chromatin immunoprecipitation (ChIP) assay. ChIP assay was performed using EZ-Magna ChIP G Chromatin Immunoprecipitation kit (Merck Millipore, Darmstadt, Germany) following the manufacturer's protocol. In brief, U373MG cells infected with Ad-p53- or Ad-LacZ at a MOI of 10 were cross-linked with $1 \%$ formaldehyde for $10 \mathrm{~min}$, washed with PBS, and lysed in nuclear lysis buffer. The lysate was then sonicated using Bioruptor UCD-200 (CosmoBio) to shear DNA to $200-1,000$ bp. Supernatant from $1 \times 10^{6}$ cells was used for each immunoprecipitation with anti-p53 antibody (OP140, Merck Millipore) or normal mouse IgG (sc-2025, Santa Cruz). Column-purified DNA was quantified by qPCR. Primer sequences are shown in Table I.

Myo-inositol (MI) assay. To prepare cell homogenate, cells were collected and suspended in PBS. Samples were sonicated for $15 \mathrm{~min}$ with a $30 \mathrm{sec}$ on/30 sec off cycle using Bioruptor UCD-200 (Cosmobio). After centrifugation at $16,000 \mathrm{x} \mathrm{g}$ for $5 \mathrm{~min}$, myo-inositol content in supernatants was measured using myo-inositol assay kit (K-INOSL, Megazyme International Ireland, Bray, Wicklow, Ireland) according to the manufacturer's instructions.

Colony formation assay. HCT116 cells and HepG2 cells were seeded on 6-well flat bottomed microplates. At $24 \mathrm{~h}$ after seeding, cells were transfected with pCAGGS (mock) vector or pCAGGS/ISYNA1 isoform 1. HCT116 and HepG2 cells were cultured with 0.5 or $1.2 \mathrm{mg} / \mathrm{ml}$ of G418, respectively. After 2 or 3 weeks of drug selection, colonies were washed in phosphatebuffered saline and stained with $0.1 \%$ crystal violet for $24 \mathrm{~h}$.

ATP assay. HCT116 $p 53^{+++}$cells were transfected with siRNAs and seeded on 24-well plates. At $24 \mathrm{~h}$ after transfection, cells were treated with $2 \mu \mathrm{g} / \mathrm{ml}$ of ADR for $2 \mathrm{~h}$. At $48 \mathrm{~h}$ after ADR treatment, cell viability was evaluated by Cell Titer-Glo Luminescent Cell Viability assay (Promega). After removal of culture medium, cells were incubated with $100 \mu \mathrm{l}$ of Cell Titer-Glo reagent and $100 \mu \mathrm{l}$ of culture medium for $10 \mathrm{~min}$ and lysed. The luminescence of cell lysate was measured by ARVO X3 plate reader (Perkin-Elmer, Waltham, MA, USA) according to the manufacturer's protocol.

Animal models. $p 53^{-1}$ mice were provided by RIKEN BioResource Center (Ibaraki, Japan) (16). The mice were 
Table I. Sequence of primers and oligonucleotides.

\begin{tabular}{lll}
\hline siRNA & \multicolumn{1}{c}{ Sense } & Antisense \\
\hline siISYNA1-A & GCGCUUCUGUGAGGUGAUUTT & AAUCACCUCACAGAAGCGCTT \\
siISYNA1-B & GCCUCAAGACCAUGUCCAUTT & AUGGACAUGGUCUUGAGGCTT \\
siISYNA1-C & UCAAGUCAGGCCAGACCAATT & UUGGUCUGGCCUGACUUGATT \\
sip53 & GACUCCAGUGGUAAUCUACTT & GUAGAUUACCACUGGAGUCTT \\
siEGFP & GCAGCACGACUUCUUCAAGTT & CUUGAAGAAGUCGUGCUGCTT
\end{tabular}

\begin{tabular}{lll}
\hline Expression vecter & \multicolumn{1}{c}{ Forward } & \multicolumn{1}{c}{ Reverse } \\
\hline ISYNA1 isoform 1 & CCCGATATCGCCGCGATGGAGGCCGCCGC & CCCCTCGAGGGTGGTGGGCATTGGGGGC \\
ISYNA1 isoform 4 & CCCGATATCCTGCCCATGGTGGCGCCC & CCCCTCGAGGGTGGTGGGCATTGGGGGC
\end{tabular}

Quantitative

Forward

Reverse

real-time PCR

\begin{tabular}{lll}
\hline hISYNA1 & AGTCCGTGCTTGTGGACTTC & CCGATAGGTTCTCCCCATC \\
hGAPDH & ACCATGGGGAAGGTGAAG & AATGAAGGGGTCATTGATGG \\
mIsyna1 & CCTTGGTGCTCCATAATACCTG & AGTCTGTGCAGAAGCTCACG \\
mGapdh & AATGTGTCCGTCGTGGATCTGA & GATGCCTGCTTCACCACCTTCT
\end{tabular}

Gene reporter assay

Forward

Reverse

RE1

RE2

CCCCTCGAGTGTATTGAGACGGGGTTTCC

CCCCTCGAGGCCTTCCTCAATGGGTCTC

CCCAAGCTTCACCCAGTCTGCTCCCTTTAAG

$\mathrm{mRE}$

CCCCTCGAGATACCCAAGTGCTGGAGGTG

CCCAAGCTTTCACGATGGACATGGTCTGTG

CCCAAGCTTACCTTGTGTGTGGTCCCTTC

Chromatin immuno-

Forward

Reverse

precipitation assay

\begin{tabular}{lcc}
\hline RE2 & GATGACTTCAAGTCAGGCCAGAC & CCCACGCACCTTGAGGCCGG \\
\hline Mutagenesis & \multicolumn{1}{c}{ Forward } & Reverse \\
\hline ISYNA1 isoform 2 & AGGCCAACTACTACGGCTCGCTGA & CCTTGAGAACGCCACCCTCGCGGC \\
RE1mt & CACTGTTCCTGGCTGACTGCCTATTTTCG & GCTAATACCTGTAATCCTAGCACTTTGGGAG \\
RE2mt & GTGTTTTTGGACTTCCTCATTGGCTCCGGC & GGAATTAACTTTGGTCTGGCCTGACTTGAAG \\
mREmt & GGGTTATTTACCACCACTGATGCCGTGACC & AGGAATACTGGCCCTGTACACCCGTGCTTG \\
\hline
\end{tabular}

maintained under specific pathogen-free conditions and were handled in accordance with the Guidelines for Animal Experiments of the University of Tokyo. $p 53^{+/+}$and $p 53^{-/-}$mice at 6 weeks of age were irradiated with $10 \mathrm{~Gy}$ of X-ray. At $24 \mathrm{~h}$ after irradiation, mice were sacrificed for liver extraction.

Database analysis. ISYNA1 expression and p53 mutation status in clinical samples were obtained from the TCGA project via data portal on 15 May 2015 (17). The association between ISYNAI expression and the presence of the $p 53$ gene mutation was determined by using the Student's t-test.

\section{Results}

p53 regulates genes related with myo-inositol metabolism. To screen novel p53 target genes, we conducted cDNA microarray analysis using mRNAs isolated from HCT116 $p 53^{+/+}$ and HCT116 $p 53^{-/-}$cells that were treated with $2 \mu \mathrm{g} / \mathrm{ml}$ of adriamycin (ADR). Fig. 1A shows a schematic representation of inositol phosphate metabolism pathway. The result of cDNA microarray analysis indicated that five genes related with myo-inositol metabolism were induced by p53 (Fig. 1B). We selected inositol 3-phosphate synthase (ISYNA1) for further analysis, because ISYNAI showed the highest expression among the five genes.

To validate the result of cDNA microarray analysis, we performed quantitative real-time PCR (qPCR) analysis and western blotting of ISYNA1 using HCT116 $p 53^{+/+}$and HCT116 $p 53^{-/-}$cells treated with ADR. As a result, we found dose-dependent induction of ISYNA1 mRNA and protein only in HCT116 $\mathrm{p53}^{+/+}$cells in response to ADR treatment (Fig. 1C). We also confirmed the induction of ISYNA1 mRNA and protein by ADR treatment in HepG2 (Fig. 1D). Moreover, transfection with siRNA against p53 remarkably inhibited 


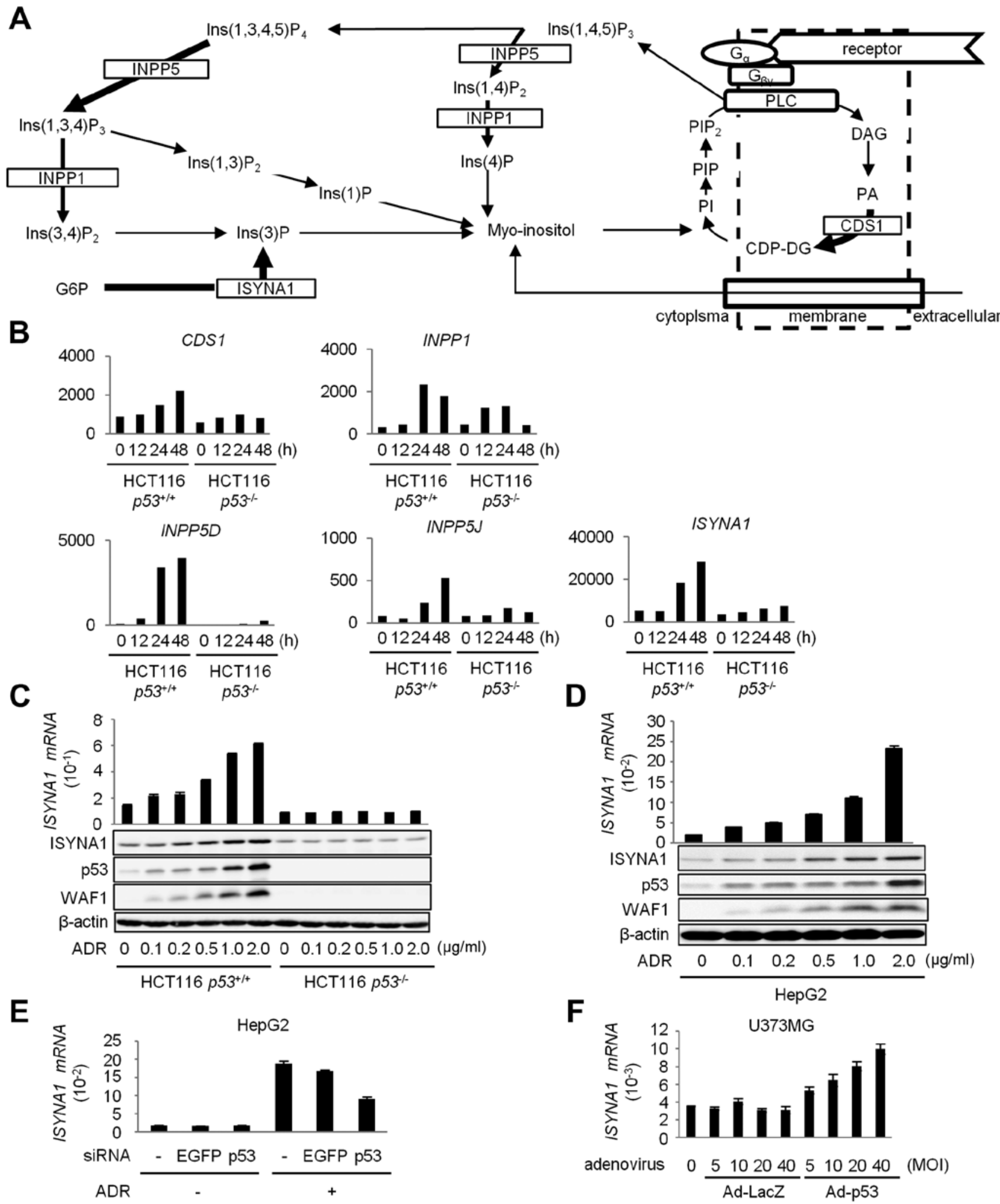

Figure 1. Regulation of ISYNA1 by p53. (A) Schematic representation of the inositol phosphate metabolism pathway. PI, phosphatidylinositol; PIP, phosphatidylinositol 4-phosphate; $\mathrm{PIP}_{2}$, phosphatidylinositol 4,5-bisphosphate; $\operatorname{Ins}(1,4,5) \mathrm{P}_{3}$, inositol 1,4,5-trisphosphate; Ins $(1,4) \mathrm{P}_{2}$, inositol 1,4-bisphosphate; Ins(4) $\mathrm{P}$, inositol 4-phosphate; Ins $(1,3,4,5) \mathrm{P}_{4}$, inositol 1,3,4,5-tetrakisphosphate; Ins $(1,3,4) \mathrm{P}_{3}$, inositol 1,3,4-trisphosphate; Ins(3,4) $\mathrm{P}_{2}$, inositol 3,4-phosphtate; Ins(3)P, inositol 3-phosphate; Ins(1,3) $\mathrm{P}_{2}$, inositol 1,3-bisphosphate; Ins(1)P, inositol 1-phosphate; G6P, glucose 6-phosphate; DAG, diacylglycerol; PA, phosphatidate; CDP-DAG, CDP-diacylglycerol. (B) Induction of genes related with myo-inositol biosynthesis by p53. HCT116 $p 53^{+/+}$and HCT116 $p 53^{-\%}$ cells were treated with $2 \mu \mathrm{g} / \mathrm{ml}$ of adriamycin (ADR) for $2 \mathrm{~h}$. mRNAs isolated from these cells were subjected to microarray analysis. Five genes related with inositol phosphate metabolism were shown to be induced by p53. (C) qPCR analysis (upper) and western blotting (lower) of ISYNA1, p53, and WAF1 in HCT116 p53 $3^{+/+}$and

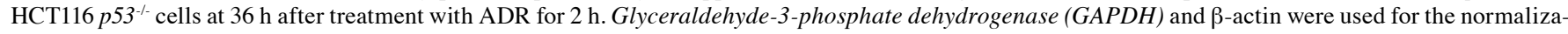
tion of expression levels. Error bars represent SD (n=3). (D) qPCR analysis (upper) and western blotting (lower) of ISYNA1, p53, and WAF1 in HepG2 cells at $36 \mathrm{~h}$ after treatment with ADR for $2 \mathrm{~h}$. GAPDH and $\beta$-actin were used for the normalization of expression levels. Error bars represent $\mathrm{SD}(\mathrm{n}=3)$. (E and F) qPCR analysis of ISYNA1 mRNA in HepG2 (E) or U373MG (F) cells. At $24 \mathrm{~h}$ after transfection of each siRNA, HepG2 cells were treated with $2 \mu \mathrm{g} / \mathrm{ml}$ of ADR for $2 \mathrm{~h}$. At $40 \mathrm{~h}$ after treatment, cells were harvested for qPCR analysis. U373MG cells were harvested at $36 \mathrm{~h}$ after infection with Ad-p53. siEGFP or Ad-LacZ were used as controls. GAPDH was used for the normalization of expression levels. Error bars represent $\mathrm{SD}(\mathrm{n}=3)$. 
A $1 \mathrm{~kb}$

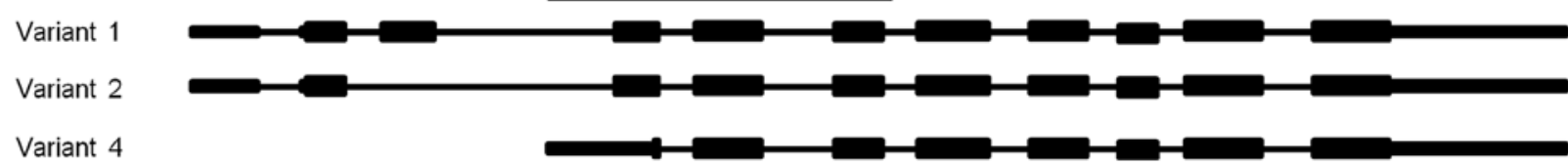

Variant 4

$$
100 \text { aa }
$$

Isoform 1

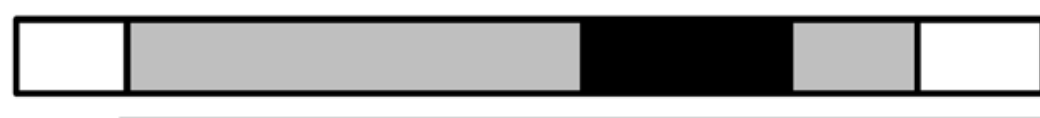

$\square$ NAD binding

5 domain

Isoform 2

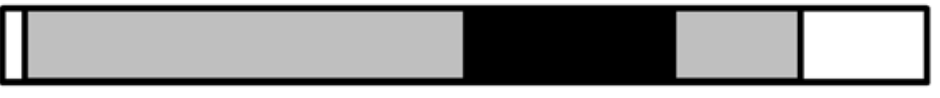

Isoform 4

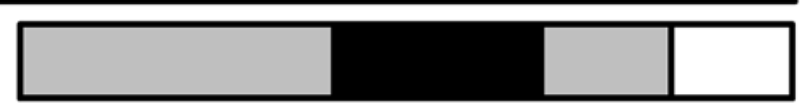

Inositol-1-phosphate synthase domain

B

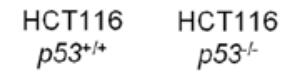

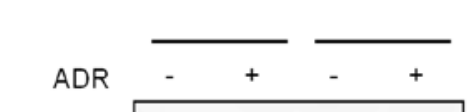

ISYNA1
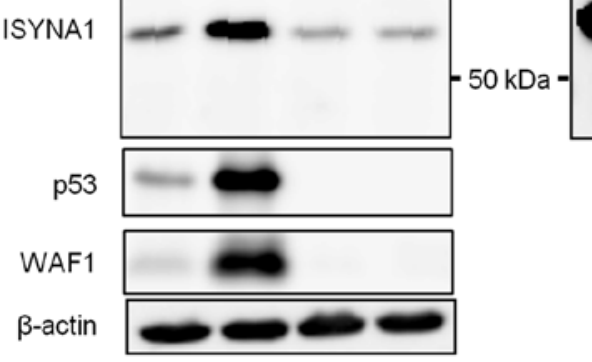

C ADR

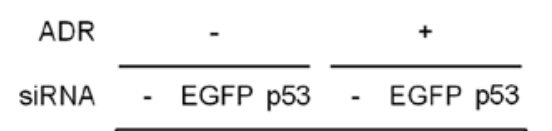

HepG2

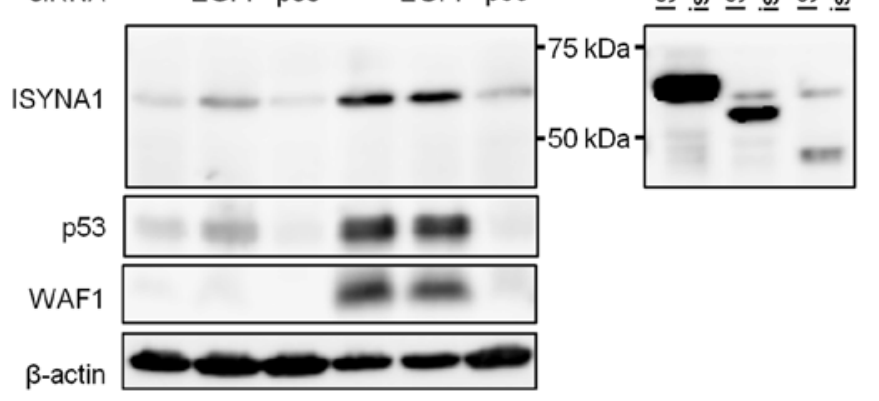

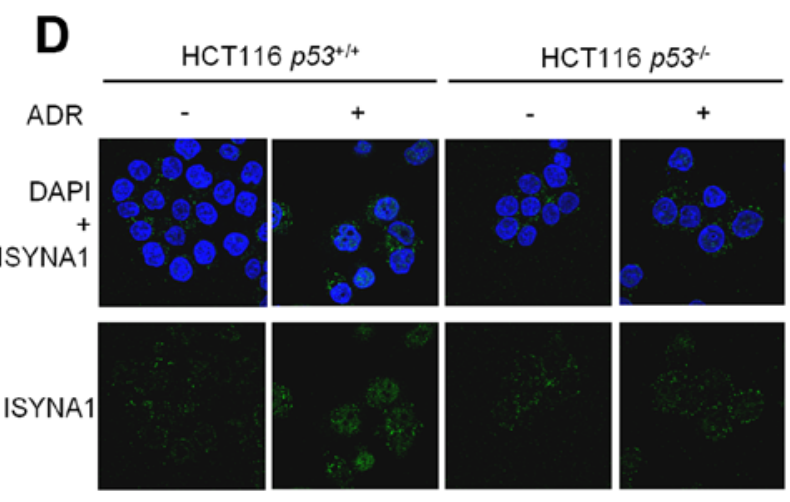

Figure 2. Expression and localization of ISYNA1. (A) Upper, genomic structure of ISYNAl variants. Black boxes indicate the locations and relative sizes of exons. Lower, domain structure of ISYNA1 isoforms. (B) Western blotting of ISYNA1, p53, and WAF1 at $36 \mathrm{~h}$ after treatment with $2 \mu \mathrm{g} / \mathrm{ml}$ of adriamycin (ADR) for $2 \mathrm{~h}$ in HCT116 $p 53^{+/+}$and HCT116 $p 53^{-/}$cells. HEK293T cells transfected with plasmid designed to express ISYNA1 isoforms 1,2 and 4 were used for molecular weight estimation of endogenous ISYNA1 protein. $\beta$-actin was used for the normalization of expression levels. (C and E) At $24 \mathrm{~h}$ after transfection of each siRNA, HepG2 cells were treated with $2 \mu \mathrm{g} / \mathrm{ml}$ of ADR for $2 \mathrm{~h}$. At $40 \mathrm{~h}$ after treatment, ISYNA1 expression was evaluated by (C) western blotting or (E) immunocytochemistry with an anti-ISYNA1 antibody (Alexa Fluor 488; green). Expression of p53, WAF1, and $\beta$-actin is also shown. DAPI was used to visualize the nuclei (blue). (D) Immunocytochemical analysis of ISYNA1 with an anti-ISYNA1 antibody (Alexa Fluor 488 ; green) at $36 \mathrm{~h}$ after treatment with $2 \mu \mathrm{g} / \mathrm{ml}$ of ADR for $2 \mathrm{~h}$ in $\mathrm{HCT} 116 p 53^{+/+}$and HCT116 $p 53^{-/}$cells. DAPI was used to visualize the nuclei (blue).

the induction of ISYNAI (Fig. 1E). p53-mediated induction of ISYNA1 was also observed in U373MG glioblastoma cells that were infected with adenovirus designed to express wild-type p53 (Ad-p53) (Fig. 1F). These results clearly indicated that ISYNA1 was regulated by $\mathrm{p} 53$.
Expression and subcellular localization of ISYNAI. There are three major variants of human ISYNA1, namely isoform 1,2, and 4. All isoforms are similar in domain structure as shown in Fig. 2A. We constructed plasmids expressing each isoform. Result of western blotting indicated that isoform 1 is the major 
A

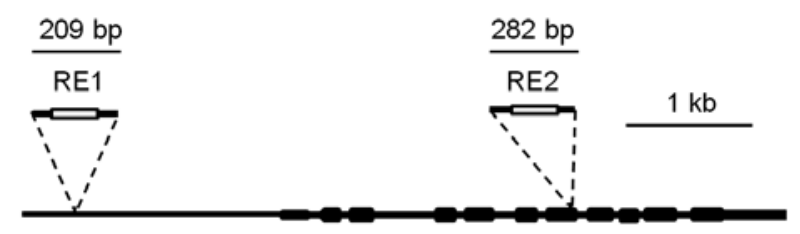

p53RE R R R $\underline{\mathcal{C} W W} \underline{G} Y Y Y R R R \underline{\mathcal{C} W W} \underline{G} Y Y Y$

RE1 A G G $\underline{C A} T \underline{G}$ a g C $\subset A \subset \underline{C g} T \underline{G C} \subset T$

RE2 A G t $\underline{C} A$ A $\underline{G} T C C G$ t $G \underline{C} T$ T $\underline{G} T g g$

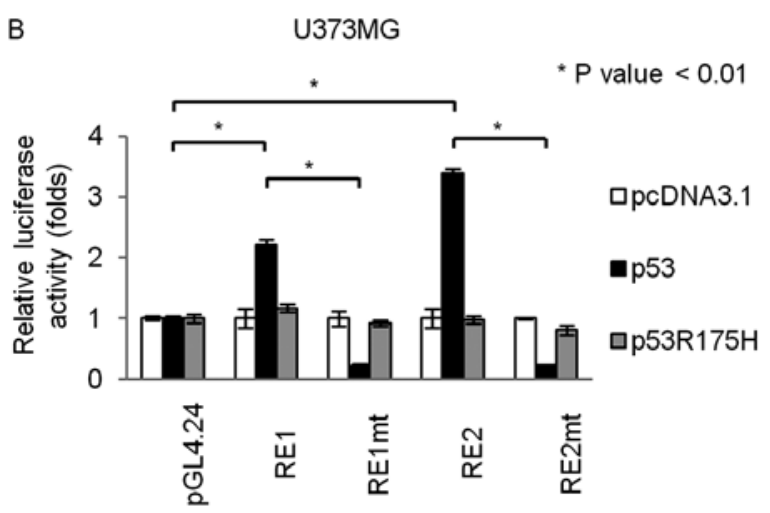

C

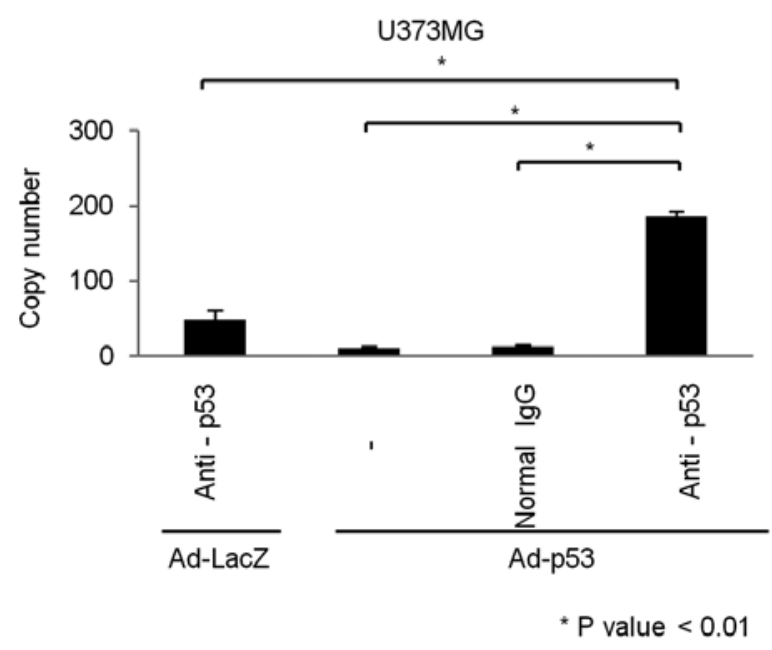

Figure 3. Identification of ISYNA1 as a novel p53 target. (A) Upper, genomic structure of human ISYNAI. Black boxes indicate the locations and relative sizes of exons. White boxes indicate the locations of p53 response elements (RE). Lower, comparison of two REs to the consensus p53 RE. $\mathrm{R}$, purine; $\mathrm{W}, \mathrm{A}$ or $\mathrm{T}$; Y pyrimidine. Identical nucleotides to the consensus sequence are written in capital letters. The underlined cytosine and guanine were substituted for thymine to examine the specificity of the p53-binding site. (B) Luciferase assay of REs with or without mutations in the RE by using U373MG cells. Luciferase activity is indicated relative to the activity of the mock vectors. The mutant p53 (R175H) was used as a negative control. Error bars represent $\mathrm{SD}(\mathrm{n}=3)$. (C) ChIP assay was performed using U373MG cells that were infected with Ad-p53 (lanes 2-4) or Ad-LacZ (lane 1) at a MOI of 10. DNA-protein complexes were immunoprecipitated with an anti-p53 antibody (lanes 1 and 4) followed by qPCR analysis. Immunoprecipitates with a normal IgG (lane 3) or in the absence of an antibody (lane 2) were used as negative controls. Error bars, $\mathrm{SD}(\mathrm{n}=3)$.

ISYNA1 isoform that was expressed in HCT116 and HepG2 cells treated with ADR (Fig. 2B and C).
Then we performed immunocytochemical analysis using HCT116 $p 53^{+/+}$, HCT116 $p 53^{-/}$cells, or HepG2 cells (Fig. 2D and E). ADR treatment increased ISYNA1 protein in the cytoplasm and the nucleus of HCT116 $p 53^{+/+}$and HepG2 cells, but ISYNA1 expression was very low in HCT116 $p 53^{-/}$cells or HepG2 cells treated with sip53.

Identification of ISYNA1 as a novel p53 target. To investigate whether ISYNA1 is a direct target of p53, we searched for p53 response element (RE) (18) within the ISYNA1 genomic region which is located on chromosome 19p13. We found putative p53 RE in the promoter region (RE1) and the seventh exon (RE2) (Fig. 3A). We subcloned DNA fragments including the RE1 or RE2 into pGL4.24 vector (pGL4.24/RE1 and pGL4.24/RE2) and performed gene reporter assay using U373MG cells. As a result, U373MG cells transfected with pGL4.24/RE1 or pGL4.24/RE2 showed enhanced luciferase activity only in the presence of plasmid expressing wild-type p53 (Fig. 3B). In addition, base substitutions within the RE1 and RE2 (pGL4.24/RE1mt and pGL4.24/RE2mt) completely abolished the enhancement of luciferase activity (Fig. 3B). To investigate whether p53 could directly bind to RE2 which showed higher transcriptional activatity, we performed chromatin immunoprecipitation (ChIP) assay using U373MG cells that were infected with Ad-p53 or Ad-LacZ. qPCR analysis of the immunoprecipitated DNA indicated that the p53 protein bound to the genomic fragment that included the RE2 (Fig. 3C). Taken together, p53 directly regulated ISYNAI expression through binding to the RE2 in the seventh exon.

Growth suppressive effect of ISYNA1. ISYNA1 is the ratelimiting enzyme of myo-inositol de novo synthesis (7) which is conserved among eukaryotes (19-25). To evaluate the biosynthesis of myo-inositol by ISYNA1, we performed myoinositol (MI) assay using HEK293T cells that were transfected with mock or plasmid expressing mock or ISYNA1 isoform 1 (Fig. 4A). The results showed that intracellular myo-inositol content in cells expressing ISYNA1 isoform 1 was significantly higher than those in control cells. In addition, DNA damage significantly increased intracellular myo-inositol content in HCT116 $p 53^{+/+}$cells, but did not affect the myo-inositol content in HCT116 $p 53^{-/}$cells (Fig. 4B). Thus, our results indicated that p53 could regulate intracellular myo-inositol levels in response to DNA damage.

We also evaluated the effect of p53-ISYNA1 pathway on cancer cell growth. The result of colony formation assay using HCT116 and HepG2 cells indicated that ISYNA1 overexpression suppressed cell proliferation (Fig. 4C). We then designed three siRNAs (siA, siB and siC) and found that siRNAs effectively suppressed ISYNA1 mRNA and protein (Fig. 4D). We performed ATP assay using HCT116 $p 53^{+/+}$cells and found that ISYNA1-silencing caused resistance to ADR treatment (Fig. 4E). These results indicated ISYNA1 is likely to be one of the key mediators of p53 induced growth suppression.

Regulation of ISYNA1 by p53 in vivo. Since ISYNA1 is conserved among eukaryotes, we investigated whether mouse Isyna1 is also regulated by p53. p53 wild-type or p53 knockout mice at 6 weeks of age were irradiated with $10 \mathrm{~Gy}$ of X-ray. At $24 \mathrm{~h}$ after irradiation, we isolated total RNA 
A

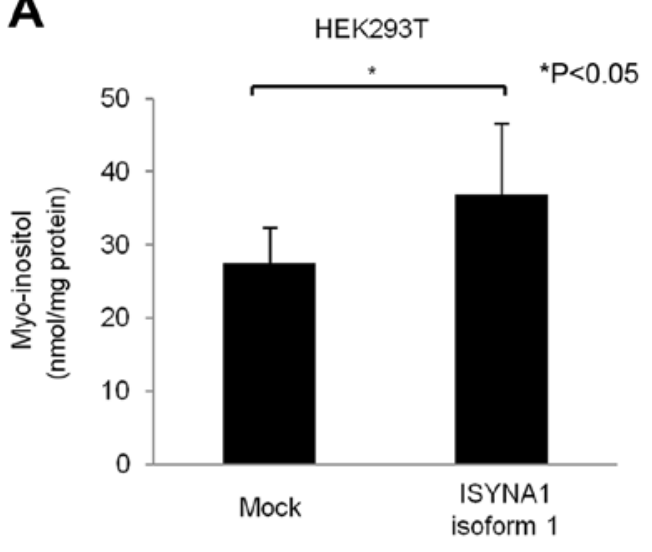

C

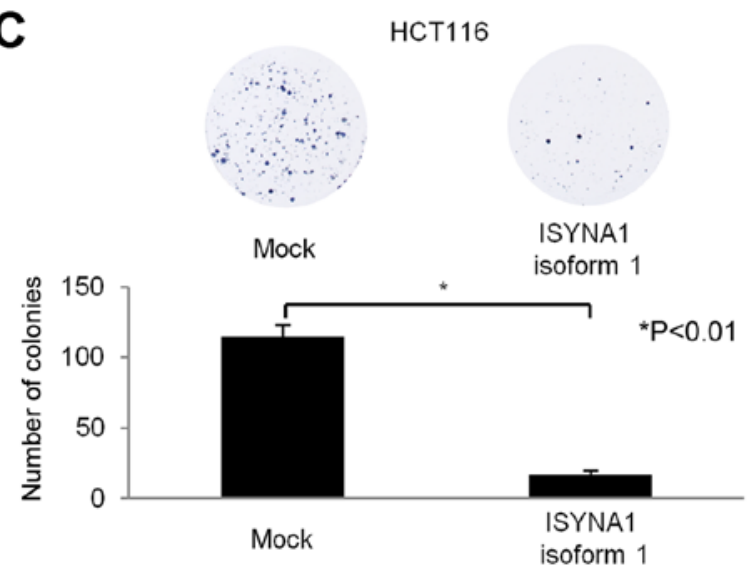

D

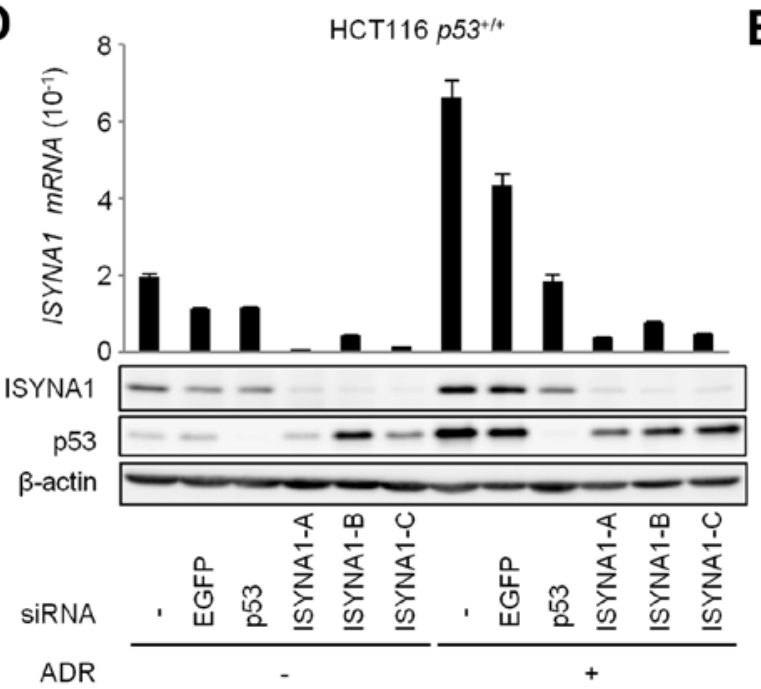

B

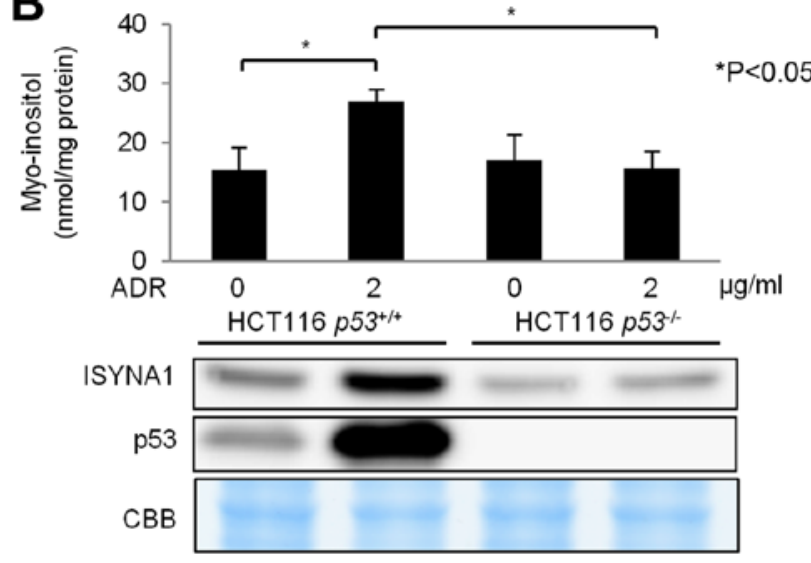

HepG2
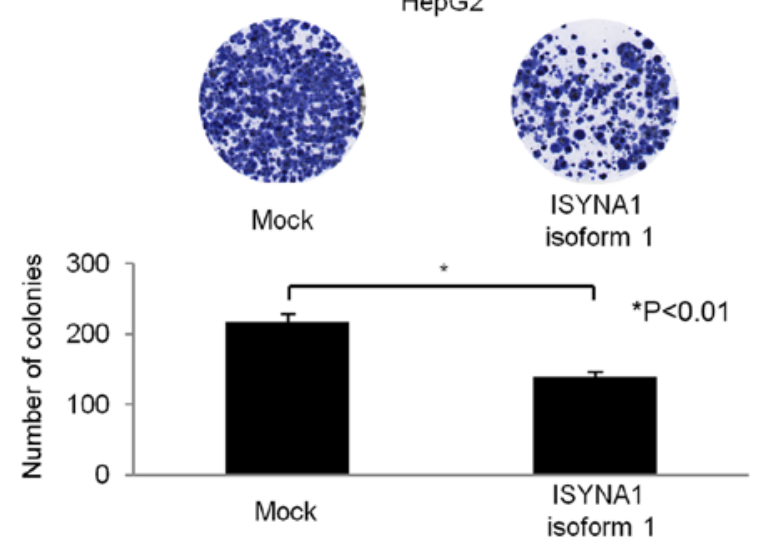

$\mathbf{E}$

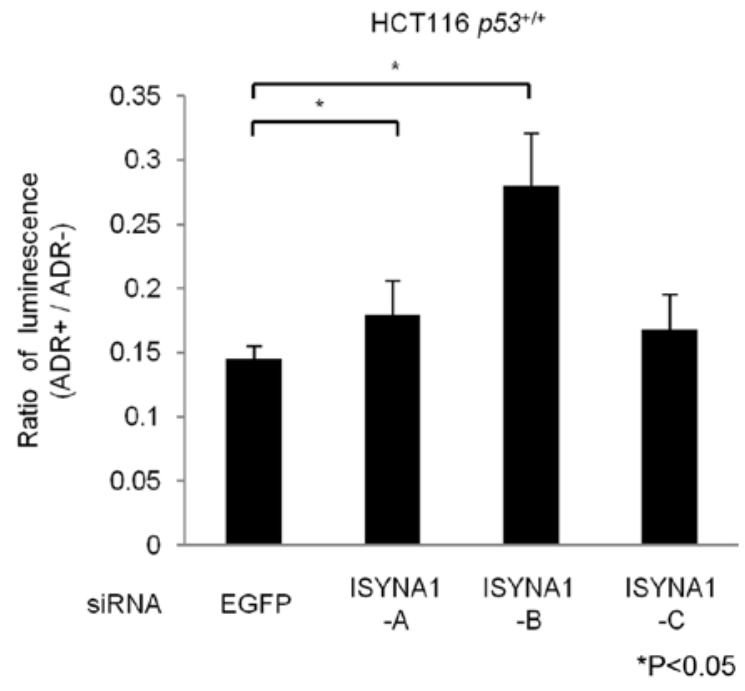

Figure 4. Regulation of myo-inositol synthesis and cell growth by p53-ISYNA1 pathway. (A) At $36 \mathrm{~h}$ after transfection with mock vector or plasmid expressing ISYNA1 isoform 1, the amounts of myo-inositol were evaluated. Total protein content was used for normalization. Error bars, SD (n=3). (B) Upper, myoinositol assay at $36 \mathrm{~h}$ after treatment with $2 \mu \mathrm{g} / \mathrm{ml}$ of ADR in HCT116 $p 53^{+/+}$and HCT116 $p 53^{-/}$cells. Total protein content was used for normalization. Error bars, SD (n=3). Lower, expression of ISYNA1 and p53 protein. (C) HCT116 and HepG2 cells were transfected with mock or plasmid expressing ISYNA1 isoform 1. The number of colonies was quantified by ImageJ software. Error bar, SD (n=3). (D) At $24 \mathrm{~h}$ after transfection of each siRNA, HCT116 $p 53^{+/+}$cells were treated with $2 \mu \mathrm{g} / \mathrm{ml}$ of ADR for $2 \mathrm{~h}$. At $48 \mathrm{~h}$ after treatment, qPCR (upper) and western blot (lower) analyses were performed to evaluate the expression of ISYNA1 and p53. siEGFP was used as a control. GAPDH and $\beta$-actin were used for the normalization of expression levels. Error bars represent SD (n=3). (E) At $24 \mathrm{~h}$ after transfection of each siRNA, HCT116 $p 53^{+/+}$cells were treated with $2 \mu \mathrm{g} / \mathrm{ml}$ of ADR for $2 \mathrm{~h}$. At $48 \mathrm{~h}$ after treatment, ATP assay was performed. Relative cell viability was calculated by dividing the luminescence of ADR-treated cells by that of untreated cells. Error bars represent $\mathrm{SD}(\mathrm{n}=3$ ).

from liver tissues. qPCR analysis revealed that mouse Isynal mRNA was induced by DNA damage only in p53 wild-type mice (Fig. 5A). Screening of p53 RE within Isynal genomic region identified a putative $\mathrm{RE}(\mathrm{mRE})$ at $\sim 10 \mathrm{~kb}$ upstream of the Isynal gene (Fig. 5B). We subcloned a DNA fragment including $\mathrm{mRE}$ into the pGL4.24 vector (pGL4.24/mRE) and performed gene reporter assay using U373MG cells (Fig. 5C). Luciferase activity was strongly enhanced by co-transfection 
A

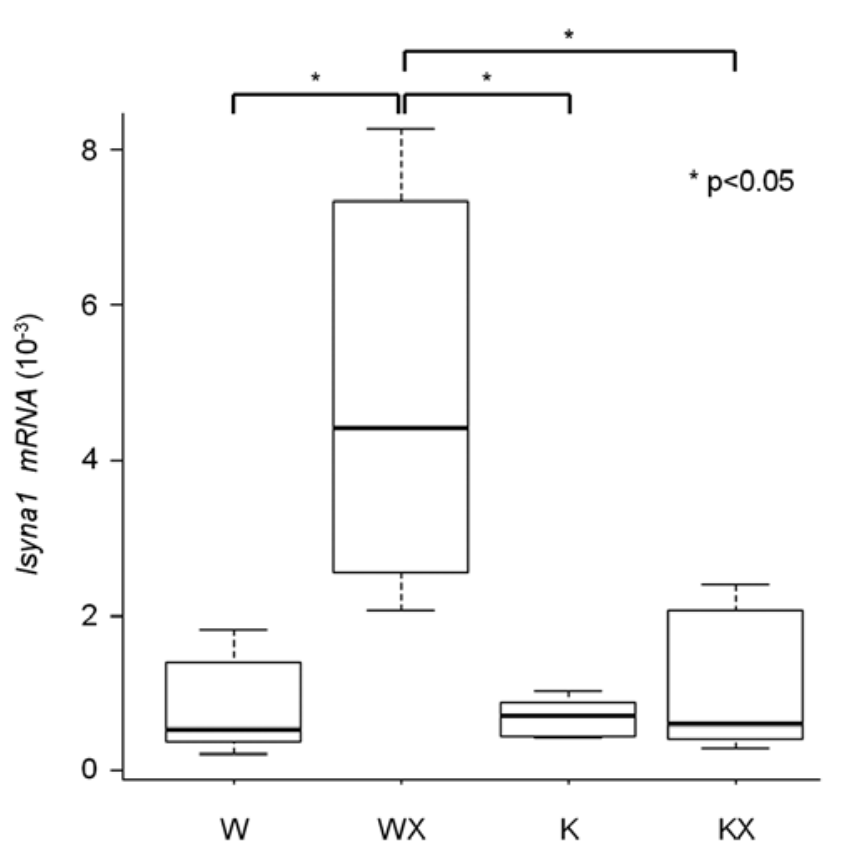

B

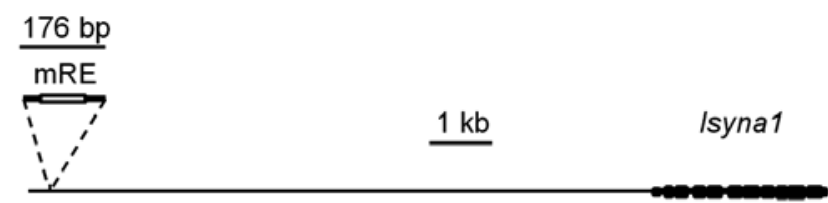

p53RE R R R $\underline{\mathrm{C} W W} \underline{\mathrm{G}} \mathrm{Y} Y \mathrm{Y} R \mathrm{R} R \underline{\mathrm{C} W W} \underline{\mathrm{G}} \mathrm{Y} Y \mathrm{Y}$ $\operatorname{mRE} \subset A G \underline{C A} T \underline{G} \subset \subset T G G G \underline{C} T A \underline{G} T T$ a C U373MG

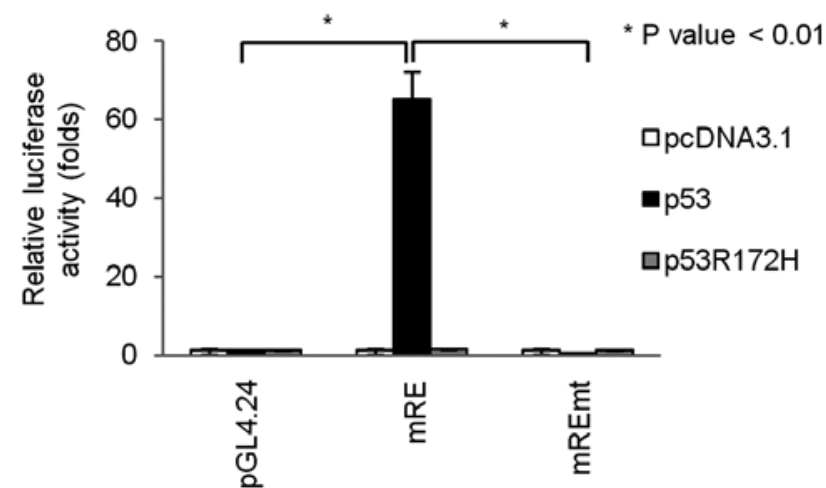

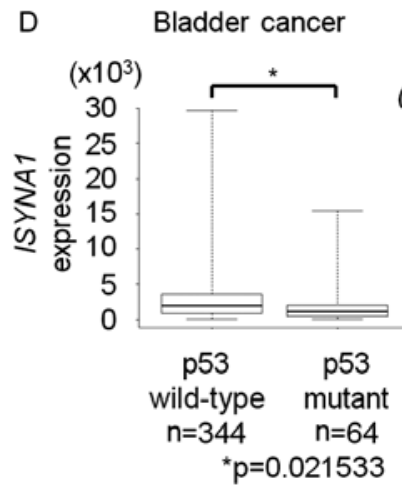

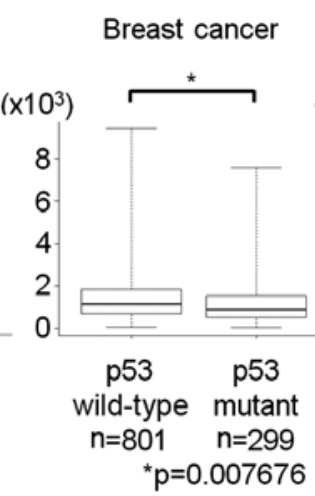

Head and neck squamous cell carcinoma squamous cell carcinoma

Pancreatic
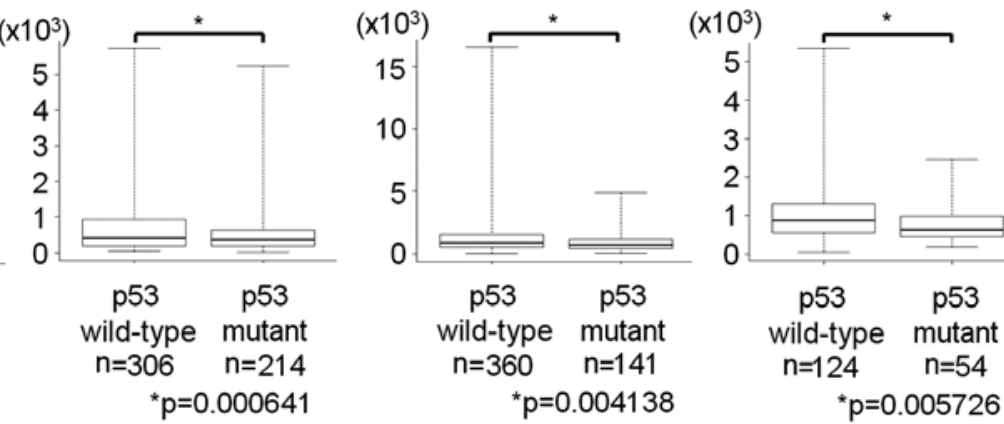

Figure 5. Regulation of ISYNA1 by p53 in vivo. (A) qPCR analysis of Isynal in mouse livers. Mice were divided into four groups; $p 53$ wild-type mice without irradiation (W), p53 wild-type mice with irradiation (WX), 553 knockout mice without irradiation $(\mathrm{K}), p 53$ knockout mice with irradiation (KX) $(\mathrm{n}=6 \mathrm{per}$ group). Glyceraldehyde-3-phosphate dehydrogenase (Gapdh) was used for the normalization of expression level. Top bar represents maximum observation, lower bar represents minimum observation, the top side of the box represents the third quartile, and the bottom side, the first quartile. The middle bar represents the median value. The P-value was calculated by Student's t-test. (B) Upper, genomic structure of mouse Isynal. Black boxes indicate the locations and relative sizes of exons. The white box indicates the location of the p53 response element (mRE). Lower, comparison of mRE to the consensus p53RE. R, purine; W, A or T; Y pyrimidine. (C) Luciferase assay of mRE in U373MG with or without mutation of the RE. Luciferase activity is indicated relative to the activity of the mock vectors. The plasmid expressing mouse p53 carrying a missense mutation $(\mathrm{R} 172 \mathrm{H})$ served as a negative control. Error bars represent the SD (n=3). (D) Box plot of ISYNA1 expression in bladder cancer, breast cancer, head and neck squamous cell carcinoma, lung squamous cell carcinoma, and pancreatic adenocarcinoma tissues from the TCGA database. The vertical axis indicates the normalized expression level of ISYNA1, top bar represents maximum observation, lower bar represents minimum observation, the top side of the box represents the third quartile, and the bottom side, the first quartile. The middle bar represents the median value. The P-value was calculated by Student's t-test.

with wild-type p53, but not with mutant p53. In addition, base substitutions within mRE diminished the enhancement of luciferase activity, demonstrating regulation of Isynal by $\mathrm{p} 53$ through mRE.

We also analyzed whether p53 regulates ISYNAI in human cancer tissues. Correlation between p53 mutation and ISYNAI expression was analyzed by using omics data of various tumor tissues released from the TCGA database (17). Interestingly, ISYNAI mRNA expression in bladder cancer, breast cancer, head and neck squamous cell carcinoma, lung squamous cell carcinoma, and pancreatic adenocarcinoma was significantly decreased in tumor tissues with p53 mutation compared with those without 553 mutation (Fig. 5D). These findings indicate that p53 regulates ISYNAI expression in vivo.

\section{Discussion}

We identified ISYNA1 as a novel p53 target. ISYNA1 is a key enzyme which affects myo-inositol de novo synthesis (7,26,27). In addition, p53 induced INPPI and INPP5 (28) that are involved in myo-inositol salvage pathway. Myo-inositol is one of the chemical compounds which is essential for living organisms (29), and myo-inositol depletion affects cell survival and growth (30). Myo-inositol was also reported to 
suppress tumor growth in vitro and in vivo (31-38). Previous studies indicated that myo-inositol suppresses phosphorylation of Akt and Erk by inhibiting PI3K activity $(12,13)$. p53 was also shown to suppress PI3K-Akt pathway by inducing PTEN (39) and Phlda3 (40). Our results suggested a novel mechanism whereby 553 negatively regulates PI3K-Akt pathway by inducing ISYNA1.

Epidemiological studies indicate that myo-inositol prevents progression of dysplasia in smokers (11-13), and decreases tumorigenesis in chronic hepatitis patients (33). These findings suggested that p53 would suppress tumorigenesis by inducing biosynthesis of myo-inositol. We also found that ISYNA1 was induced in mouse liver tissue by DNA damage. To evaluate the chemopreventive effect of myo-inositol, we fed p53 knockout mice with myo-inositol in drinking water. However, oral myo-inositol did not suppress tumor development (data not shown). Although, myo-inositol was shown to suppress liver cancer $(32,33)$, liver cancer is relatively rare for p53 knockout mice compared with lymphoma of thymus or spleen (41). In addition, although induction of Isynal was observed in liver tissues, Isynal was not induced in thymus and spleen (data not shown). Therefore, to evaluate the chemopreventive effect of myo-inositol or ISYNAI in vivo, liver cancer model would be appropriate.

Taken together, ISYNA1 was shown to be a mediator of p53-dependent growth suppression, and ISYNAI expression was reduced in several types of cancers with p53 mutations. Therefore, myo-inositol could be a potential anticancer agent for cancer cells with p53 mutation. Our findings revealed a novel role of p53 in myo-inositol biosynthesis which could be a possible therapeutic target.

\section{Acknowledgements}

We thank Satomi Takahashi and Misato Oshima for technical assistance. We also thank The Cancer Genome Atlas (TCGA) project and members of the Cancer Genomics Hub (CGHub) for making all TCGA data publicly accessible. This study was supported partially by grant from Japan Society for the Promotion of Science and Ministry of Education, Culture, Sports, Science and Technology of Japan to K.M and C.T., grant from Japan Agency for medical Research and Development to K.M. and C. T., grant from the Ministry of Health, Labour and Welfare, Japan to K.M., and grant in-Aid from the Tokyo Biochemical Research Foundation to K.M.

\section{References}

1. Brady CA and Attardi LD: p53 at a glance. J Cell Sci 123 2527-2532, 2010.

2. Levine AJ and Oren M: The first 30 years of p53: Growing ever more complex. Nat Rev Cancer 9: 749-758, 2009.

3. Hirao A, Kong YY, Matsuoka S, Wakeham A, Ruland J, Yoshida H, Liu D, Elledge SJ and Mak TW: DNA damageinduced activation of $\mathrm{p} 53$ by the checkpoint kinase Chk 2 . Science 287: 1824-1827, 2000.

4. Bensaad K, Tsuruta A, Selak MA, Vidal MN, Nakano K, Bartrons R, Gottlieb E and Vousden KH: TIGAR, a p53-inducible regulator of glycolysis and apoptosis. Cell 126: 107-120, 2006.

5. Hu W, Zhang C, Wu R, Sun Y, Levine A and Feng Z: Glutaminase 2, a novel p53 target gene regulating energy metabolism and antioxidant function. Proc Natl Acad Sci USA 107: 7455-7460, 2010
6. Kenzelmann Broz D, Spano Mello S, Bieging KT, Jiang D, Dusek RL, Brady CA, Sidow A and Attardi LD: Global genomic profiling reveals an extensive p53-regulated autophagy program contributing to key p53 responses. Genes Dev 27: 1016-1031, 2013.

7. Ju S, Shaltiel G, Shamir A, Agam G and Greenberg ML: Human 1-D-myo-inositol-3-phosphate synthase is functional in yeast. J Biol Chem 279: 21759-21765, 2004.

8. Croze ML and Soulage CO: Potential role and therapeutic interests of myo-inositol in metabolic diseases. Biochimie 95: 1811-1827, 2013.

9. Maeba R, Hara H, Ishikawa H, Hayashi S, Yoshimura N, Kusano J, Takeoka Y, Yasuda D, Okazaki T, Kinoshita M, et al: Myo-inositol treatment increases serum plasmalogens and decreases small dense LDL, particularly in hyperlipidemic subjects with metabolic syndrome. J Nutr Sci Vitaminol (Tokyo) 54: 196-202, 2008

10. Mukai T, Kishi T, Matsuda Y and Iwata N: A meta-analysis of inositol for depression and anxiety disorders. Hum Psychopharmacol 29: 55-63, 2014.

11. Lam S, McWilliams A, LeRiche J, MacAulay C, Wattenberg L and Szabo E: A phase I study of myo-inositol for lung cancer chemoprevention. Cancer Epidemiol Biomarkers Prev 15: 1526-1531, 2006

12. Han W, Gills JJ, Memmott RM, Lam S and Dennis PA: The chemopreventive agent myoinositol inhibits Akt and extracellular signal-regulated kinase in bronchial lesions from heavy smokers. Cancer Prev Res (Phila) 2: 370-376, 2009.

13. Gustafson AM, Soldi R, Anderlind C, Scholand MB, Qian J, Zhang X, Cooper K, Walker D, McWilliams A, Liu G, et al: Airway PI3K pathway activation is an early and reversible event in lung cancer development. Sci Transl Med 2: 26ra25, 2010.

14. Oda K, Arakawa H, Tanaka T, Matsuda K, Tanikawa C, Mori T, Nishimori H, Tamai K, Tokino T, Nakamura Y, et al: p53AIP1, a potential mediator of p53-dependent apoptosis, and its regulation by Ser-46-phosphorylated p53. Cell 102: 849-862, 2000.

15. Tanikawa C, Matsuda K, Fukuda S, Nakamura Y and Arakawa H: p53RDL1 regulates p53-dependent apoptosis. Nat Cell Biol 5: 216-223, 2003.

16. Tsukada T, Tomooka Y, Takai S, Ueda Y, Nishikawa S, Yagi T, Tokunaga T, Takeda N, Suda Y, Abe S, et al: Enhanced proliferative potential in culture of cells from p53-deficient mice. Oncogene 8: 3313-3322, 1993.

17. Taura M, Eguma A, Suico MA, Shuto T, Koga T, Komatsu K, Komune T, Sato T, Saya H, Li JD, et al: p53 regulates Toll-like receptor 3 expression and function in human epithelial cell lines. Mol Cell Biol 28: 6557-6567, 2008.

18. el-Deiry WS, Kern SE, Pietenpol JA, Kinzler KW and Vogelstein B: Definition of a consensus binding site for p53. Nat Genet 1: 45-49, 1992.

19. Michell RH: Inositol derivatives: Evolution and functions. Nat Rev Mol Cell Biol 9: 151-161, 2008.

20. Seelan RS, Lakshmanan J, Casanova MF and Parthasarathy RN: Identification of myo-inositol-3-phosphate synthase isoforms: Characterization, expression, and putative role of a $16-\mathrm{kDa}$ gamma(c) isoform. J Biol Chem 284: 9443-9457, 2009.

21. Konarzewska P, Esposito $\mathrm{M}$ and Shen $\mathrm{CH}$ : INO1 induction requires chromatin remodelers Ino80p and Snf2p but not the histone acetylases. Biochem Biophys Res Commun 418: 483-488, 2012.

22. Valluru R and Van den Ende W: Myo-inositol and beyond emerging networks under stress. Plant Sci 181: 387-400, 2011.

23. Deranieh RM, He Q, Caruso JA and Greenberg ML: Phosphorylation regulates myo-inositol-3-phosphate synthase: A novel regulatory mechanism of inositol biosynthesis. J Biol Chem 288: 26822-26833, 2013.

24. Parthasarathy RN, Lakshmanan J, Thangavel M, Seelan RS, Stagner JI, Janckila AJ, Vadnal RE, Casanova MF and Parthasarathy LK: Rat brain myo-inositol 3-phosphate synthase is a phosphoprotein. Mol Cell Biochem 378: 83-89, 2013.

25. Henry SA, Gaspar ML and Jesch SA: The response to inositol: Regulation of glycerolipid metabolism and stress response signaling in yeast. Chem Phys Lipids 180: 23-43, 2014.

26. Guan G, Dai P and Shechter I: cDNA cloning and gene expression analysis of human myo-inositol 1-phosphate synthase. Arch Biochem Biophys 417: 251-259, 2003.

27. Chauvin TR and Griswold MD: Characterization of the expression and regulation of genes necessary for myo-inositol biosynthesis and transport in the seminiferous epithelium. Biol Reprod 70: 744-751, 2004. 
28. Ye Y, Jin L, Wilmott JS, Hu WL, Yosufi B, Thorne RF, Liu T, Rizos H, Yan XG, Dong L, et al: PI(4,5)P2 5-phosphatase A regulates $\mathrm{PI} 3 \mathrm{~K} / \mathrm{Akt}$ signalling and has a tumour suppressive role in human melanoma. Nat Commun 4: 1508, 2013.

29. Ohnishi T, Murata T, Watanabe A, Hida A, Ohba H, Iwayama Y, Mishima K, Gondo Y and Yoshikawa T: Defective craniofacial development and brain function in a mouse model for depletion of intracellular inositol synthesis. J Biol Chem 289: 10785-10796, 2014.

30. Eagle H, Oyama VI, Levy M and Freeman AE: Myo-inositol as an essential growth factor for normal and malignant human cells in tissue culture. J Biol Chem 226: 191-205, 1957.

31. Hecht SS, Upadhyaya P, Wang M, Bliss RL, McIntee EJ and Kenney PM: Inhibition of lung tumorigenesis in A/J mice by $\mathrm{N}$-acetyl-S-(N-2-phenethylthiocarbamoyl)-L-cysteine and myoinositol, individually and in combination. Carcinogenesis 23 : 1455-1461, 2002

32. Lee HJ, Lee SA and Choi H: Dietary administration of inositol and/or inositol-6-phosphate prevents chemically-induced rat hepatocarcinogenesis. Asian Pac J Cancer Prev 6: 41-47, 2005.

33. Nishino H: Phytochemicals in hepatocellular cancer prevention Nutr Cancer 61: 789-791, 2009.

34. Vucenik I and Shamsuddin AM: Protection against cancer by dietary IP6 and inositol. Nutr Cancer 55: 109-125, 2006.

35. Wattenberg LW and Estensen RD: Chemopreventive effects of myo-inositol and dexamethasone on benzo[a]pyrene and 4-(methylnitrosoamino)-1-(3-pyridyl)-1-butanone-induced pulmonary carcinogenesis in female A/J mice. Cancer Res 56: 5132-5135, 1996.
36. Witschi H, Espiritu I and Uyeminami D: Chemoprevention of tobacco smoke-induced lung tumors in $\mathrm{A} / \mathrm{J}$ strain mice with dietary myo-inositol and dexamethasone. Carcinogenesis 20: 1375-1378, 1999.

37. Kassie F, Kalscheuer S, Matise I, Ma L, Melkamu T, Upadhyaya P and Hecht SS: Inhibition of vinyl carbamate-induced pulmonary adenocarcinoma by indole-3-carbinol and myo-inositol in A/J mice. Carcinogenesis 31: 239-245, 2010.

38. Kassie F, Matise I, Negia M, Lahti D, Pan Y, Scherber R, Upadhyaya P and Hecht SS: Combinations of N-Acetyl-S-(N-2Phenethylthiocarbamoyl)-L-Cysteine and myo-inositol inhibit tobacco carcinogen-induced lung adenocarcinoma in mice. Cancer Prev Res (Phila) 1: 285-297, 2008

39. Memmott RM and Dennis PA: The role of the Akt/mTOR pathway in tobacco carcinogen-induced lung tumorigenesis. Clin Cancer Res 16: 4-10, 2010.

40. Kawase T, Ohki R, Shibata T, Tsutsumi S, Kamimura N, Inazawa J, Ohta $\mathrm{T}$, Ichikawa $\mathrm{H}$, Aburatani $\mathrm{H}$, Tashiro $\mathrm{F}$, et al: PH domain-only protein PHLDA3 is a p53-regulated repressor of Akt. Cell 136: 535-550, 2009.

41. Jacks T, Remington L, Williams BO, Schmitt EM, Halachmi S, Bronson RT and Weinberg RA: Tumor spectrum analysis in p53-mutant mice. Curr Biol 4: 1-7, 1994. 